\title{
Fabrication and Characterization of Luminescence Film Sensor for Detecting Defects of Barrier Films
}

\author{
Pu-Reun Choi, ${ }^{1}$ Hyun Chul Kim, ${ }^{1}$ Sun Min Kim, ${ }^{1,2}$ and Eunhae Koo ${ }^{1}$ \\ ${ }^{1}$ Electronic Convergence Materials Division, Nano Convergence Materials Center, \\ Korea Institute of Ceramic Engineering and Technology (KICET), Jinju 52851, Republic of Korea \\ ${ }^{2}$ School of Advanced Materials and Engineering, Sungkyunkwan University, Suwon 16419, Republic of Korea \\ Correspondence should be addressed to Eunhae Koo; ehkoo@kicet.re.kr
}

Received 20 June 2016; Revised 4 October 2016; Accepted 9 October 2016

Academic Editor: Xuping Sun

Copyright (C) $2016 \mathrm{Pu}$-Reun Choi et al. This is an open access article distributed under the Creative Commons Attribution License, which permits unrestricted use, distribution, and reproduction in any medium, provided the original work is properly cited.

\begin{abstract}
The most critical issue on flexible electronics such as organic solar-cell, OLEDs, and flexible display is the protection of the core active materials from the degradation by water and oxygen. The defect of barrier film is the main channel for the transmission of water and oxygen molecules. Herein, in order to monitor the defects of barrier films, we have developed anthracene boronic acid pinacol ester (ABAPE) sensor which is very sensitive to water vapor. When ABAPE film is exposed to water, it gives off fluorescence emission at 389 and $408 \mathrm{~nm}$ under excitation peak at $366 \mathrm{~nm}$. Based on the fluorescence microscopy and SEM images, the optical method using the ABAPE sensor film can monitor the defects of barrier film smaller than $1 \mu \mathrm{m}$. This result suggests that ABAPE can be utilized to monitor the defect and water vapor transmission rate (WVTR) in the barrier film.
\end{abstract}

\section{Introduction}

Flexible electronics should be bending, rugged, and rollable without loss of their properties such as optical properties, electrical properties, and mechanical properties [1-4]. However, plastics cannot be used as a substrate for flexible electronics because they cannot protect the degradation of active materials by the reaction with water or oxygen. As oxygen and water can penetrate and diffuse quickly through polymer film, $99 \%$ of electroluminescence (EL) can be lost in $150 \mathrm{~min}$ $[5,6]$. In order to achieve lifetimes of tens of thousands of hours, water vapor transmission rate (WVTR) must be under $10^{-6} \mathrm{~g} / \mathrm{m}^{2} /$ day, and oxygen transmission rates (OTR) must be under $10^{-3} \mathrm{~cm}^{3} / \mathrm{m}^{2} /$ day. To meet the requirements, the high performance barrier film has been developed to block water vapor and oxygen to penetrate the device. The defect of barrier film, such as pinholes, line defects, and cracks, is the main channel for the transmission of water and oxygen molecules. Therefore, reducing the defect in the barrier film is crucial to enhance the barrier property [710]. Many previous studies reported the relationship between the water vapor transmission rate and the defect density of the films [11-16]. The water transmission volume $(Q)$ can be determined by (1), where $A$ is the total area of defects, $D$ is the diffusion coefficient of water molecules in the film, $L$ is the film thickness, $\phi$ is the moisture concentration on the film surface, $S$ is the total film area, and $N$ is the number of defects [15].

$$
\begin{aligned}
Q & =\frac{A \cdot D \cdot S \cdot P}{L}=\frac{A \cdot D \cdot \phi}{L}, \\
\text { WVTR } & =\sum_{0}^{N} N\left(\frac{Q}{S}\right) .
\end{aligned}
$$

The water vapor transmission rate can be obtained by (2) using the total film area $(S)$ and number of defects $(N)$. This analytic equation predicts that the defect density is linear to the water vapor transmission rate (WVTR). Recently, various image optical systems have been introduced to monitor small-sized defects. For example, the Coherence Correlation Interferometer (CCI) and the Wavelength Scanning Interferometry (WSI) were utilized to measure the density of micronscale defects, in which 100 150 defects of $3 \mu \mathrm{m}$ diameter were present per unit area $\left(\mathrm{mm}^{2}\right)$. However, because CCI and WSI 


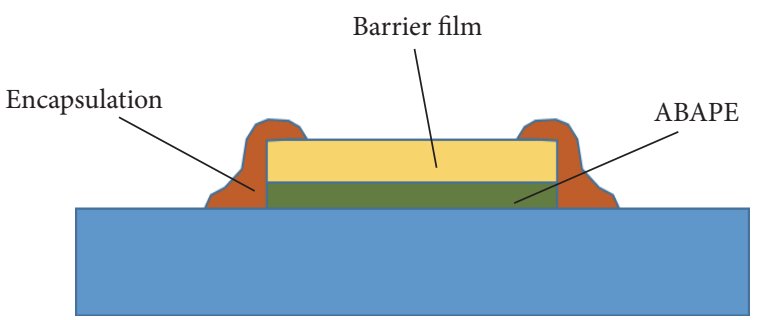

(a)

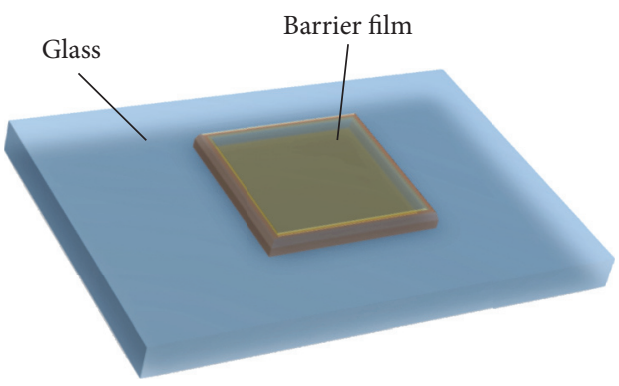

(b)

FIGURE 1: The ABAPE coated barrier film which is encapsulated in the glove box, (a) side view and (b) upper view.

system are so sophisticated to measure the defect density in the barrier film, a simple and convenient measurement system should be developed [14].

In this study, we investigate high sensitive water film sensor to monitor the film defects. Anthracene boronic acid pinacol ester (ABAPE) increases its fluorescence properties, which is very sensitive to water. When water is added, amino group of ABAPE was protonated. Emission peak appears at $389 \mathrm{~nm}$ and $408 \mathrm{~nm}$ under $366 \mathrm{~nm}$ UV lights [8]. ABAPE was dissolved in 1,2-dichloroethane (DCE) to evaluate water sensitivity. Photoluminescence spectra and water sensitivity were characterized by using a photoluminescence spectrophotometer (Sinco S-3100, Korea). The film surface has been investigated and characterized using the fluorescence microscope and the optical microscope.

\section{Experimental}

2.1. The Synthesis of Anthracene Boronic Acid Pinacol Ester (ABAPE). Sodium hydride (60\%), 9-(methylaminomethyl)anthracene, 2-bromomethylphenylboronic acid pinacol ester, $\mathrm{N}, \mathrm{N}$-dimethylmethanamide (DMF), dichloroethane (DCE), and methanol were purchased from Sigma Co. (USA, http://www.sigmaaldrich.com/). And silica gel (60 N, spherical, neutral) was purchased from Kanto Chemical. All other reagents were analytical grade and used as received. Triplex distilled water was used in the experiment.

Sodium hydride $(60 \%, 2.1 \mathrm{mmol})$ was added, after 9(methylaminomethyl) anthracene $(0.84 \mathrm{mmol})$ was dissolved in DMF $(70 \mathrm{~mL})$. The solution was stirred for $1 \mathrm{~h}$ at room temperature. 2-Bromomethylphenylboronic acid pinacol ester $(3.37 \mathrm{mmol})$ was added and the solution was stirred at room temperature for $1 \mathrm{~h}$. After concentration under pressure, the resulting residue was dissolved in dichloroethane and washed with water. The residue was chromatographed on silica gel (dichloromethane: methanol $=10: 1$ as eluent).

\subsection{Characterization of Water Sensitivity of ABAPE Solution.} The sensitivity of ABAPE to water was characterized by using a fluorescence spectrometer (FluoroMate FS-2, Scinco Co., Seoul, Republic of Korea). First, $2 \times 10^{-5}, 2 \times 10^{-6}, 2 \times 10^{-7}$, and $2 \times 10^{-8} \mathrm{~mol}$ concentrations of ABAPE were prepared using anhydrous dichloroethane. Di-water in anhydrous ethanol $(10 \%, v / v)$ stock solution was prepared to control small quantity of water. $0.5 \mu \mathrm{L}$ of anhydrous ethanol stock solution was added in $2.5 \mathrm{~mL}$ of ABAPE in DCE solution using micro syringe. The prepared water solution was in the range of 20 to $160 \mathrm{ppm}$. Dilution effect by the repetition of water injection is ignored.

2.3. Fabrication and Characterization of Barrier Films with Defects. A barrier film was fabricated to analyze the defects generated in the inorganic layer. The defects are specially studied after mechanical loading. First, a PET film substrate (SKC, thickness $100 \mu \mathrm{m}$ ) was cleaned by ultrasonication and then dried. To minimize the residual stress inside the PET film, the film was annealed at $100^{\circ} \mathrm{C}$ for $1 \mathrm{~h}$. And then to fabricate an undercoat layer, epoxy resin was coated by spin-coating. Finally, $100 \mathrm{~nm}$ of alumina layer $\left(\mathrm{Al}_{2} \mathrm{O}_{3}\right)$ was deposited by RF magnetron sputtering.

The defects in the barrier film were investigated by using the fluorescence microscopy and SEM. In order to prepare the test coupon, first, ABAPE coated layer was spin-coated onto the $\mathrm{Al}_{2} \mathrm{O}_{3}$ layer of the barrier film. ABAPE solution was prepared with $1 \mathrm{wt} \%$ of polyethylene oxide (PEO) mixed with $20 \mathrm{mM}$ of ABAPE in dichloroethane as a solvent. Finally, to prevent from penetrating water molecules in the air to ABAPE layer, the film was encapsulated with a sealant (Nagase 5570) in the glove box, as shown in Figure 1.

\section{Results and Discussion}

The sensing mechanism of water molecule by ABAPE is as shown in Figure 2. Without water, lone-pair electron of nitrogen atoms quenches the fluorescence property of anthracene moiety. When water is added, Lewis acid and electron donor of nitrogen atom dissociate water molecules. And then, zwitter ionic structure is formed. As a result, lone-pair of nitrogen atom cannot proceeds photo-induced electron transfer to quench the fluorescence characteristics of anthracene moiety. As a result, anthracene moiety gives off fluorescence emission whose emission peaks are shown at 389 and $408 \mathrm{~nm}$ under excitation peak at $366 \mathrm{~nm}$ [17-20].

Figure 3 shows the water sensitivity of ABAPE solution which indicates an increase of PL intensity as a function of the added amount of water in the solution. Generally, PL intensity increases linearly when tens of ppm of water is added in ABAPE solution. For instance, the intensity in $0.2 \mu \mathrm{M}$ ABAPE 


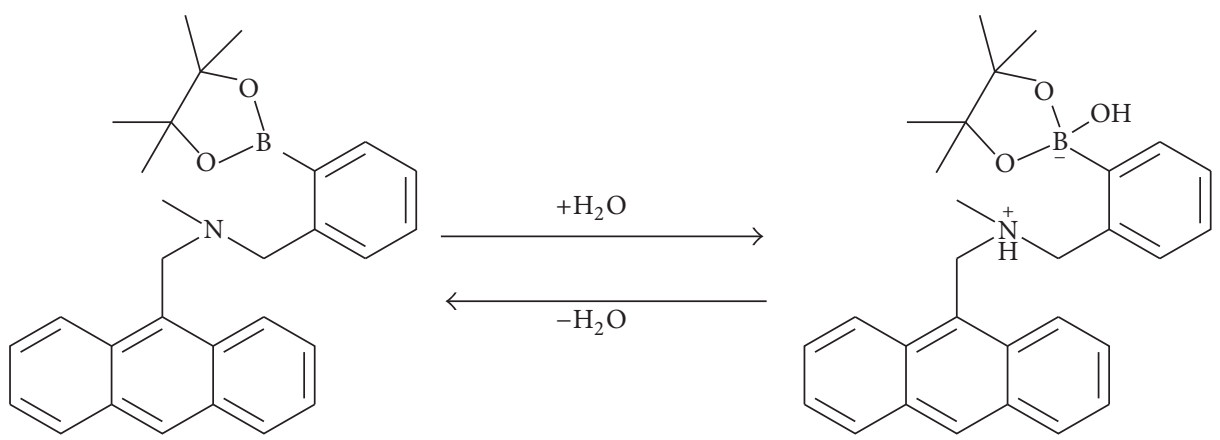

Figure 2: Water sensing mechanism of ABAPE.

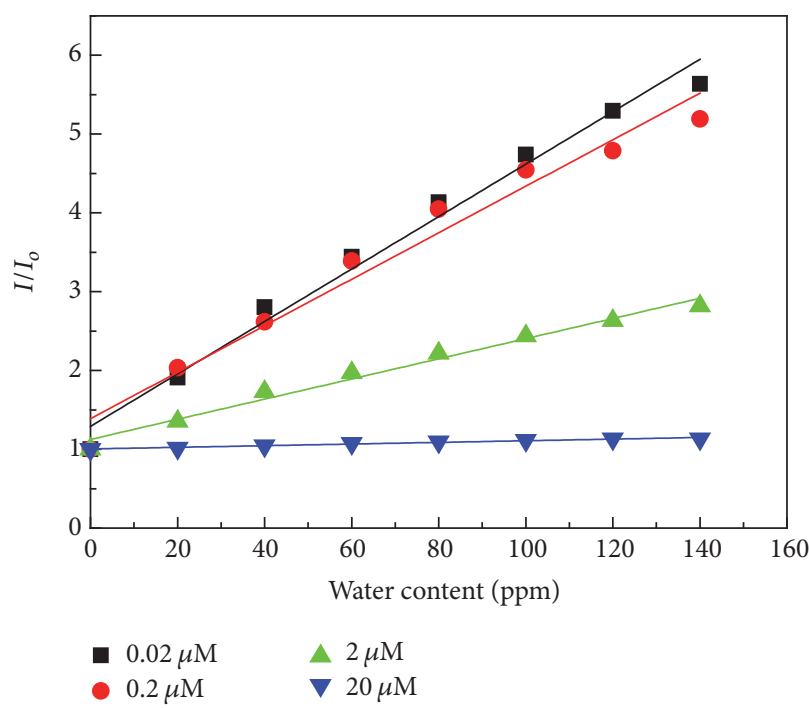

FIGURE 3: PL intensity as a function of the added amount of water in the solution.

solution increases 5 times when $7 \times 10^{-3} \mathrm{ppm}$ of water is added. In contrast, the intensity in $20 \mu \mathrm{M}$ ABAPE solution is increasing to $10 \%$ because added water is too low in the solution. In other words, the lower the ABAPE concentration in the solution is, the more a trace of water reacts effectively.

In order to investigate the defect of the barrier film, the film was deformed with $25 \mathrm{kN}$ of tensile stress by using the tensile testing machine. Figure 4 shows the stress/strain curve of the barrier film according to the different elongation percentage. In the range of the tensile strain smaller than $6 \%$, the film shows elastic deformation. However, the film shows the plastic deformation, when the film is deformed irreversibly over $6 \%$. The fluorescent signal from the ABAPE molecules is observed. In addition, the defects are observed by using a fluorescent microscope when being exposed to water through the defects in the films. Figures 5 and 6 show the fluorescence microscopy image and SEM image of barrier film coated with ABAPE solution after the film was drawn with the different tensile strain. The films with $3 \%, 8 \%$, and $22 \%$ of the tensile strain were prepared and characterized. $3 \%$ elongated film shows the maximum tensile stress of $67 \mathrm{MPa}$, but no defect lines. This suggests that the film is in the range of the elastic region, but no plastic damage in the film. In contrast, $8 \%$ and $22 \%$ tensile elongated films show many line defects which are the evidence of the plastic deformation of the films. The defect has developed in the vertical direction of the applied force. The gap between the defects is estimated to be about $50 \mu \mathrm{m}$ with the thickness of $1 \mu \mathrm{m}$. In case of the $22 \%$ tensile strained film, the number of defects was getting more, but the gap between defects was getting smaller.

Figure 7 indicates the defect density and the fluorescence intensity change of ABAPE coated barrier films after the films are exposed to water vapor in the air for 1 day. The defect density is determined by the ratio of the defect area to the total area based on the fluorescence microscopic images as estimated using Image J program. As shown in Figure 7, the defect density and the fluorescence intensity of the $22 \%$ elongated film are much larger than that of $8 \%$ elongated film. It should be noted that the defect density and fluorescence intensity of the $3 \%$ elongated film are not changed because no defect is observed in the film. These results suggest that the optical method using the ABAPE sensor film can be utilized to monitor the defect in the barrier film. 

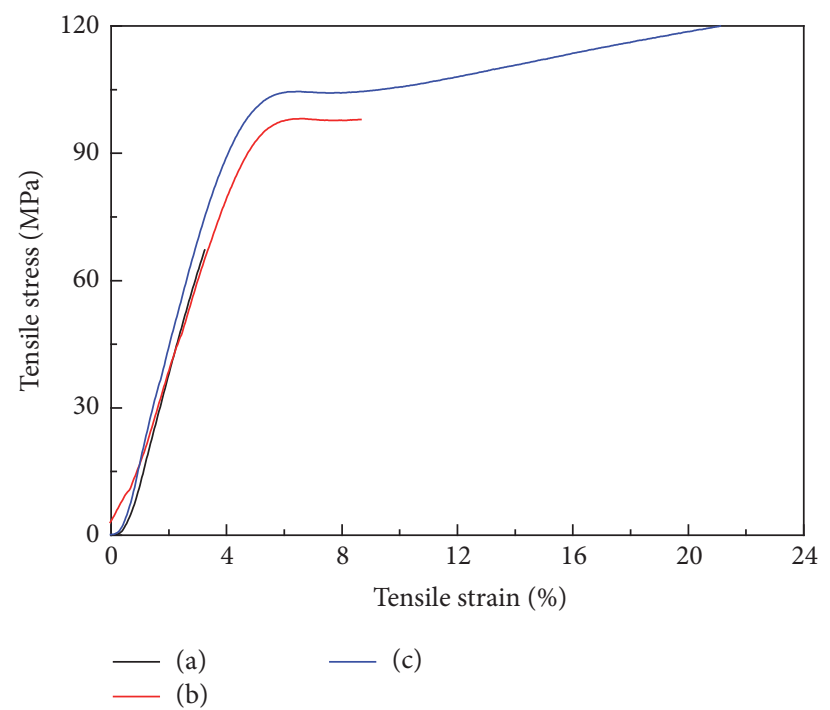

FIGURE 4: Stress-strain curve of the ABAPE coated barrier film as a function of the tensile elongation. (a) $3 \%$, (b) $8 \%$, and (c) $22 \%$.

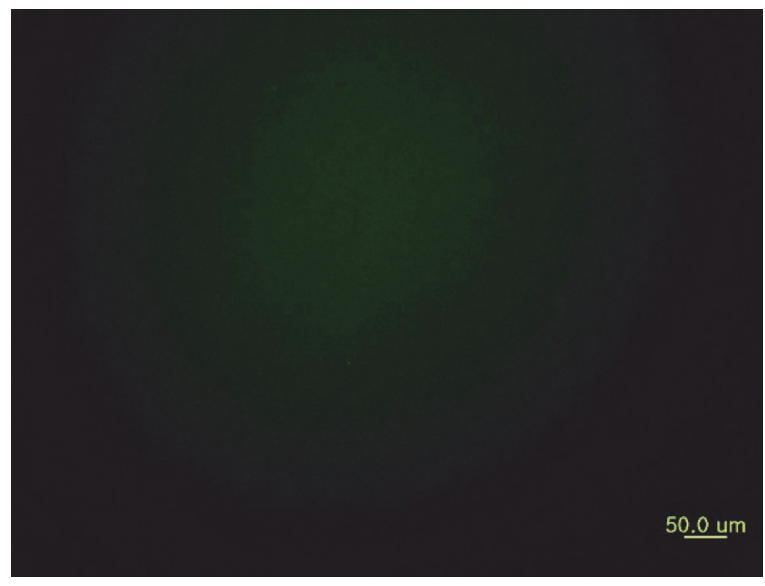

(a)

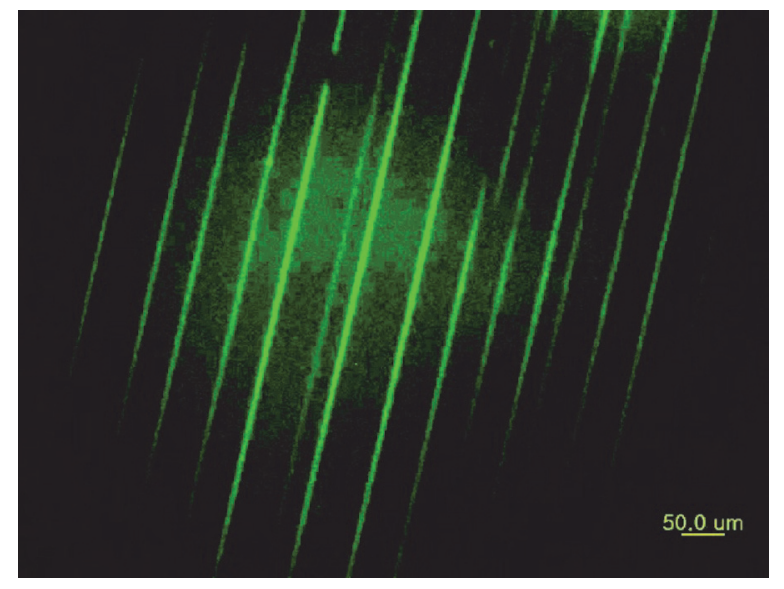

(b)

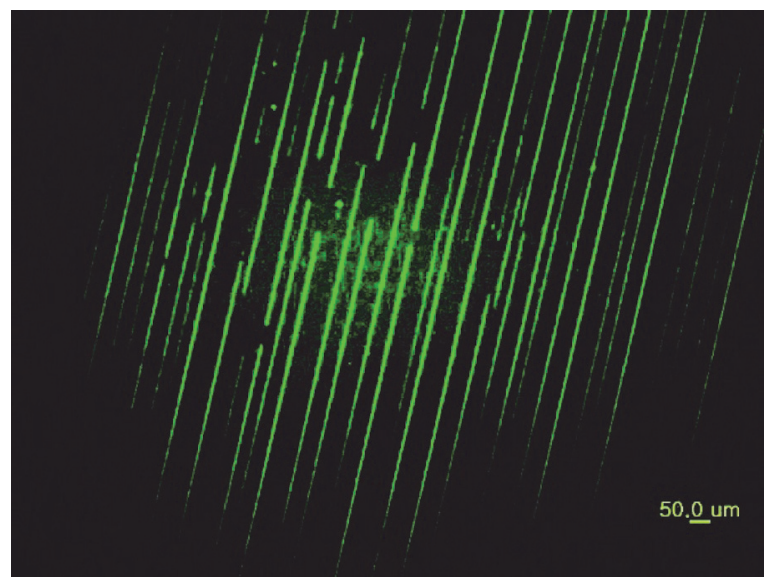

(c)

FIGURE 5: The fluorescence microscopy image of the barrier film coated with ABAPE solution as a function of the tensile elongation. (a) 3\%, (b) $8 \%$, and (c) $22 \%$. 


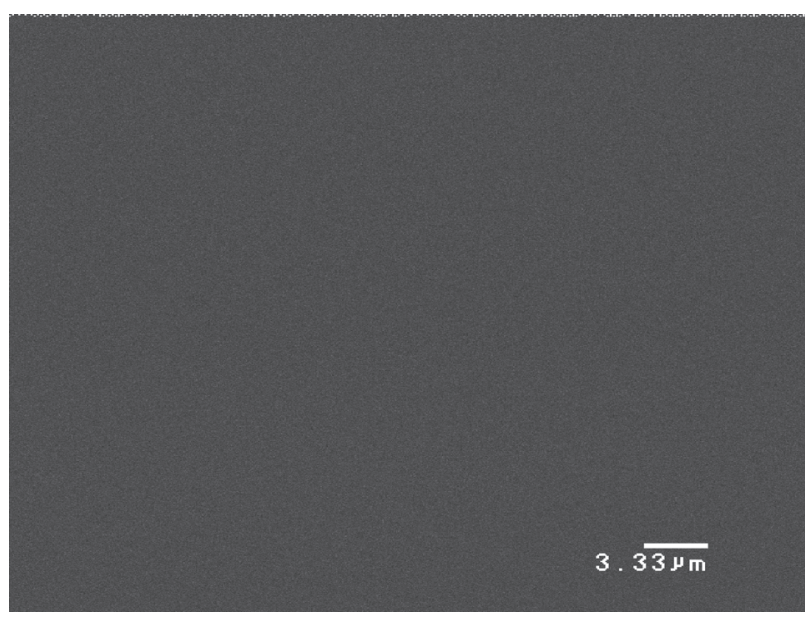

(a)

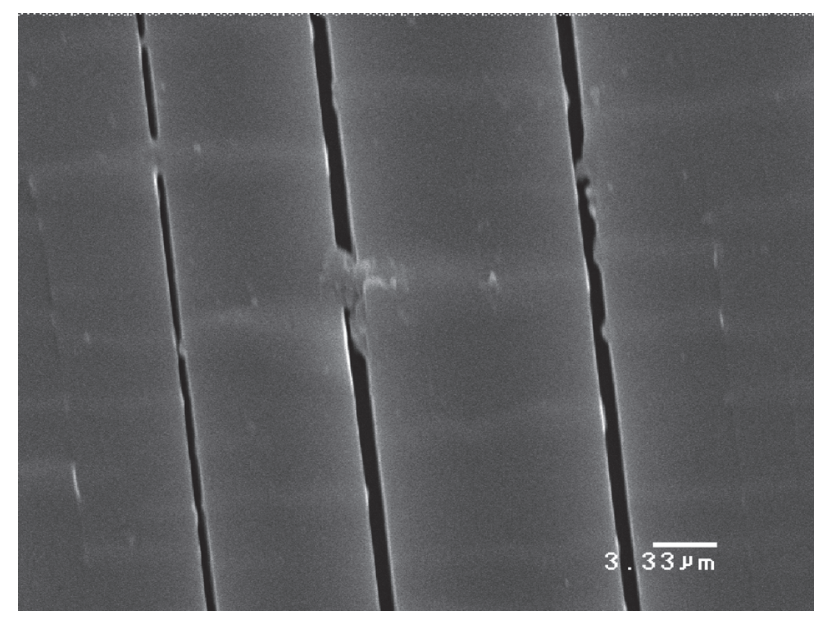

(b)

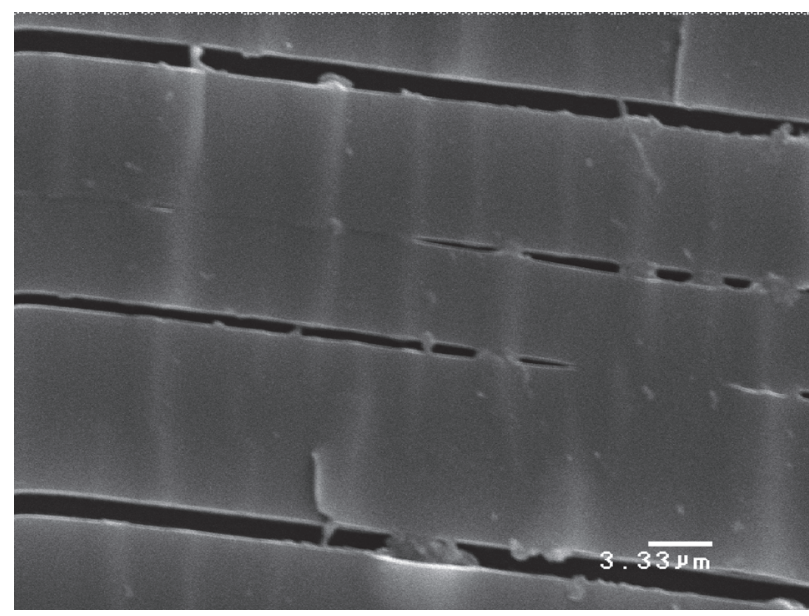

(c)

FIGURE 6: SEM image of the barrier film coated with ABAPE solution as a function of the tensile elongation. (a) $3 \%$, (b) $8 \%$, and (c) $22 \%$.

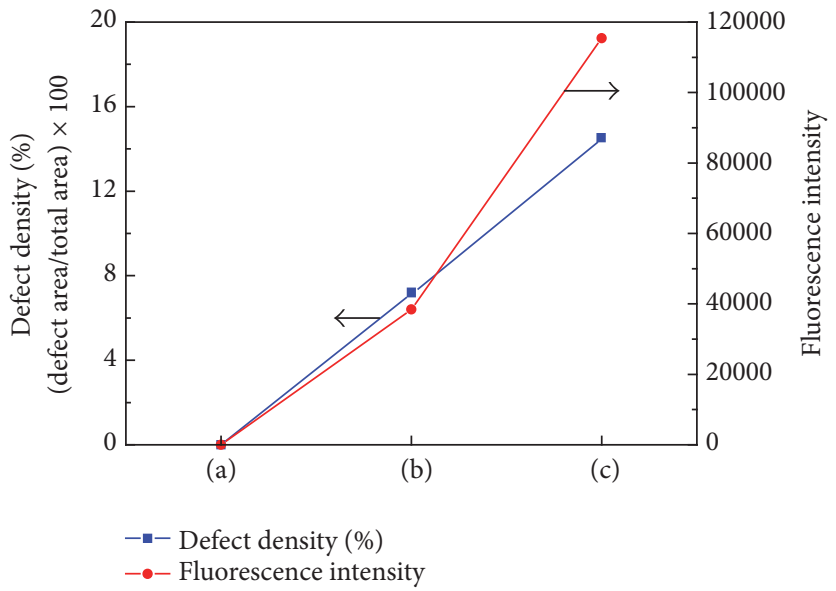

FIGURE 7: The defect density and the fluorescence intensity change of ABAPE coated barrier films after the films are exposed to water vapor. (a) $3 \%$, (b) $8 \%$, and (c) $22 \%$ tensile elongated film. 


\section{Conclusion}

In this study, we investigate a sensitive film sensor to monitor the defects of barrier films. Anthracene boronic acid pinacol ester (ABAPE) increases its fluorescence properties, which is very sensitive to water vapor. When ABAPE film is exposed to water vapor, it gives off fluorescence emission at 389 and $408 \mathrm{~nm}$ under excitation peak at $366 \mathrm{~nm}$. Based on the fluorescence microscopy and SEM image, the optical method using the ABAPE sensor film can monitor the defects of barrier film smaller than $1 \mu \mathrm{m}$. This result suggests that ABAPE can be utilized to monitor the defect in the barrier film.

\section{Competing Interests}

The authors declare that they have no competing interests.

\section{Acknowledgments}

This work was supported by a grant (Grant no. 20153010012110) from the New and Renewable Energy and a grant (Grant no. 10037859) from the Fundamental R\&D Program for Technology of World Premier Materials funded by the Ministry of Industry, Trade and Energy, Republic of Korea.

\section{References}

[1] K. Song, J. Noh, T. Jun, Y. Jung, H.-Y. Kang, and J. Moon, "Fully flexible solution-deposited $\mathrm{ZnO}$ thin-film transistors," Advanced Materials, vol. 22, no. 38, pp. 4308-4312, 2010.

[2] T. Sekitani, U. Zschieschang, H. Klauk, and T. Someya, "Flexible organic transistors and circuits with extreme bending stability," Nature Materials, vol. 9, no. 12, pp. 1015-1022, 2010.

[3] G. Gu, P. E. Burrows, S. Venkatesh, S. R. Forrest, and M. E. Thompson, "Vacuum-deposited, nonpolymeric flexible organic light-emitting devices," Optics Letters, vol. 22, no. 3, pp. 172-174, 1997.

[4] C.-J. Chiang, C. Winscom, S. Bull, and A. Monkman, "Mechanical modeling of flexible OLED devices," Organic Electronics, vol. 10, no. 7, pp. 1268-1274, 2009.

[5] D. Kolosov, D. S. English, V. Bulovic, P. F. Barbara, S. R. Forrest, and M. E. Thompson, "Direct observation of structural changes in organic light emitting devices during degradation," Journal of Applied Physics, vol. 90, no. 7, pp. 3242-3247, 2001.

[6] P. E. Burrows, V. Bulovic, S. R. Forrest, L. S. Sapochak, D. M. McCarty, and M. E. Thompson, "Reliability and degradation of organic light emitting devices," Applied Physics Letters, vol. 65, no. 23, pp. 2922-2924, 1994.

[7] G. L. Graff, R. E. Williford, and P. E. Burrows, "Mechanisms of vapor permeation through multilayer barrier films: lag time versus equilibrium permeation," Journal of Applied Physics, vol. 96, no. 4, pp. 1840-1849, 2004.

[8] W. H. Koo, S. M. Jeong, S. H. Choi, H. K. Baik, S. M. Lee, and S. J. Lee, "Water vapor barrier properties of transparent $\mathrm{SnO}_{2}-\mathrm{SiO}_{x}$ composite films on polymer substrate," The Journal of Physical Chemistry B, vol. 108, no. 49, pp. 18884-18889, 2004.
[9] V. Dimitrov and T. Komatsu, "Classification of simple oxides: a polarizability approach," Journal of Solid State Chemistry, vol. 163, no. 1, pp. 100-112, 2002.

[10] S. Takeda, M. Fukawa, Y. Hayashi, and K. Matsumoto, "Surface $\mathrm{OH}$ group governing adsorption properties of metal oxide films," Thin Solid Films, vol. 339, no. 1-2, pp. 220-224, 1999.

[11] L. Blunt, M. Elrawemi, L. Fleming, and F. Sweeney, "Correlation of micro and nano-scale defects with WVTR for aluminium oxide barrier coatings for flexible photovoltaic modules," International Journal of Precision Technology, vol. 3, no. 3, pp. 290302, 2013.

[12] H. Klumbies, P. Schmidt, M. Hähnel et al., "Thickness dependent barrier performance of permeation barriers made from atomic layer deposited alumina for organic devices," Organic Electronics, vol. 17, pp. 138-143, 2015.

[13] A. S. da Silva Sobrinho, G. Czeremuszkin, M. Latrèche, and M. R. Wertheimer, "Defect-permeation correlation for ultrathin transparent barrier coatings on polymers," Journal of Vacuum Science \& Technology A, vol. 18, pp. 149-157, 2000.

[14] M. Elrawemi, L. Blunt, H. Muhamedsalih, F. Gao, and L. Fleming, "Implementation of in process surface metrology for R2R flexible PV barrier films," International Journal of Automation Technology, vol. 9, no. 3, pp. 312-321, 2015.

[15] L. Blunt and M. Elrawemi, "Functional modelling of water vapour transmission through surface defects using surface segmentation analysis," in Proceedings of the 15th International Conference on Metrology and Properties of Engineering Surfaces, Charlotte, NC, USA, March 2015.

[16] T. Maindron, B. Aventurier, A. Ghazouani et al., "Investigation of $\mathrm{Al}_{2} \mathrm{O}_{3}$ barrier film properties made by atomic layer deposition onto fluorescent tris-(8-hydroxyquinoline) aluminium molecular films," Thin Solid Films, vol. 548, pp. 517-525, 2013.

[17] Y. Ooyama, A. Matsugasako, K. Oka et al., "Fluorescence PET (photo-induced electron transfer) sensors for water based on anthracene-boronic acid ester," Chemical Communications, vol. 47, no. 15, pp. 4448-4450, 2011.

[18] A. Prasanna de Silva, B. McCaughan, B. O. F. McKinney, and M. Querol, "Newer optical-based molecular devices from older coordination chemistry," Dalton Transactions, no. 10, pp. 19021913,2003

[19] J. F. Callan, A. P. De Silva, and D. C. Magri, "Luminescent sensors and switches in the early 21st century," Tetrahedron, vol. 61, no. 36, pp. 8551-8588, 2005.

[20] T. Gunnlaugsson, M. Glynn, G. M. Tocci (née Hussey), P. E. Kruger, and F. M. Pfeffer, "Anion recognition and sensing in organic and aqueous media using luminescent and colorimetric sensors," Coordination Chemistry Reviews, vol. 250, no. 23-24, pp. 3094-3117, 2006. 

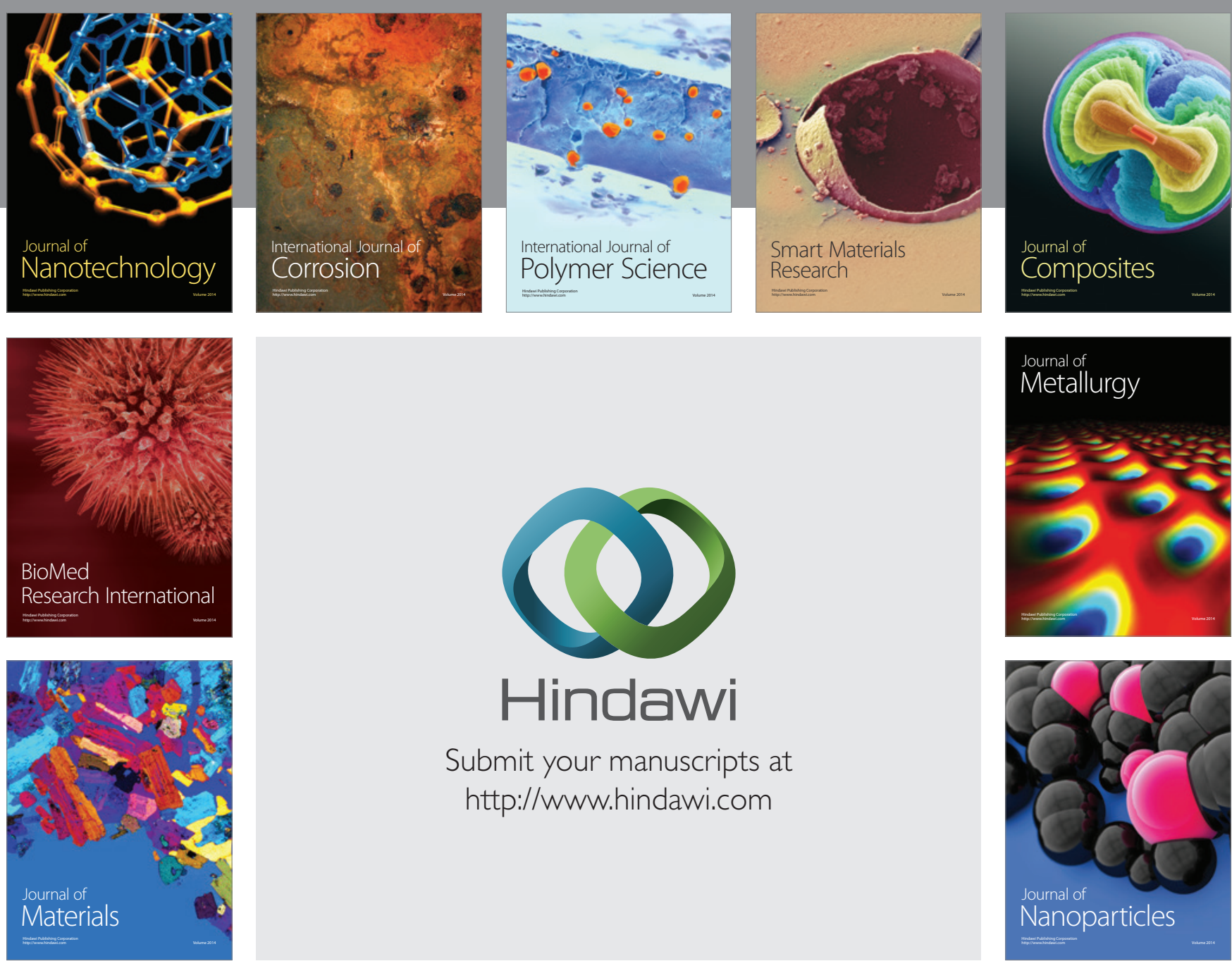

\section{Hindawi}

Submit your manuscripts at

http://www.hindawi.com

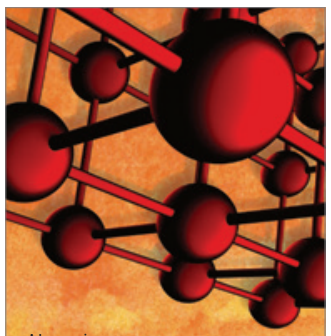

Materials Science and Engineering
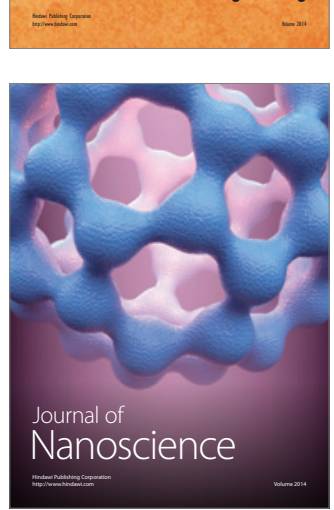
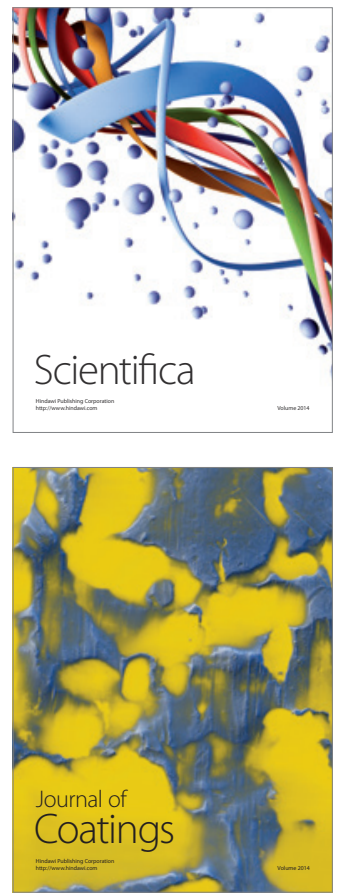
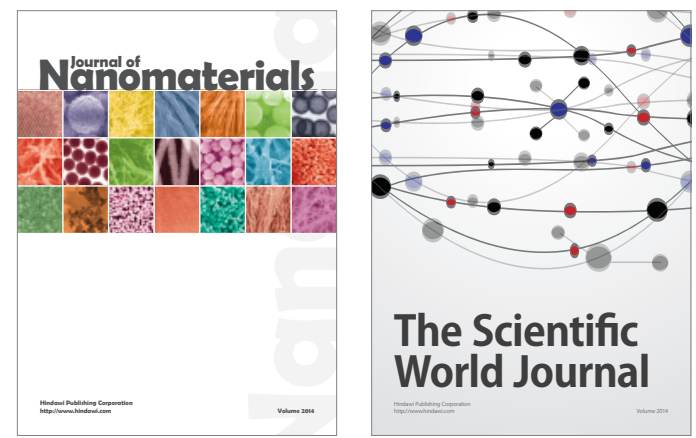

The Scientific World Journal
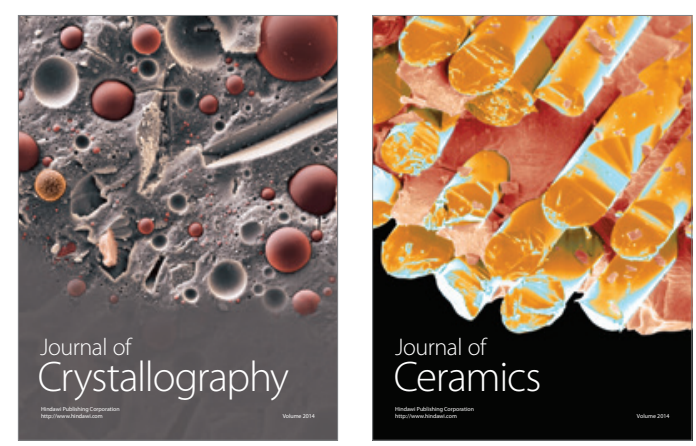
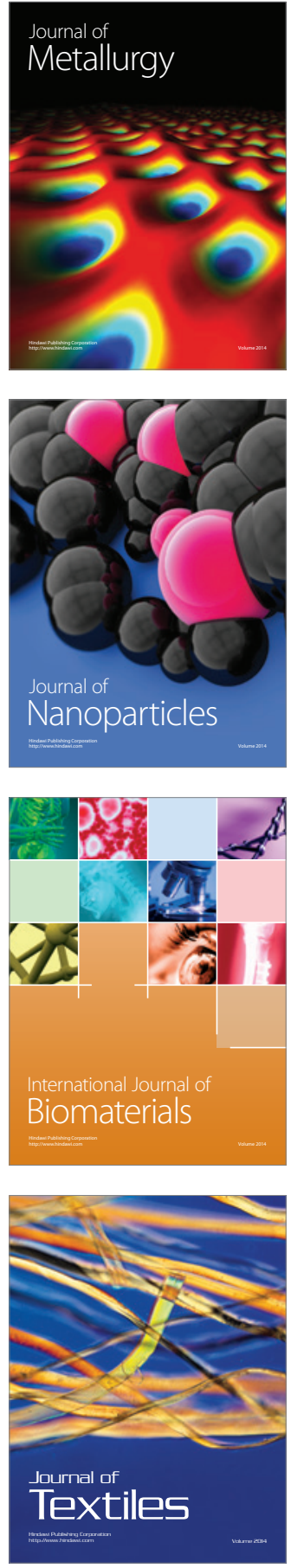